\title{
Cerebral arachnoid cysts in children
}

\author{
M. J. G. HARRISON ${ }^{1}$ \\ From the Department of Neurosurgery, The Hospital for Sick Children, Great Ormond Street, London
}

SUMMARY The case histories of 14 children are described in which hydrocephalus was found on $\stackrel{\overrightarrow{\vec{F}}}{\stackrel{\vec{S}}{+}}$ investigation to be associated with a cyst of the posterior fossa or subarachnoid cisterns. The neuroradiological and histological findings are described. The cysts are considered to be develop- $\frac{\bar{\sigma}}{\bar{\omega}}$ mental in origin. Their recognition and management are discussed.

Arachnoid cysts have been described in a variety of intracranial sites. Most reports have dealt with cysts in the Sylvian fissure (Starkman, Brown, and Linell, 1958; Anderson and Landing, 1966; Jakubiak, Dunsmore, and Beckett, 1968). Less frequent but also well recognized are cysts in the posterior fossa (Trowbridge and French, 1952; Alvord and Marcuse, 1962; Matson, 1969). Cysts around the base of the brain, and in the region of the subarachnoid cisterns are uncommon (Matson, 1969), although occasional reports have dealt with cysts in the chiasmatic region (Dott and Gillingham, 1958), in the quadrigeminal plate cistern (Alexander, 1953; Kruyff, 1965), and in the cisterna ambiens (Katagiri, 1960).

There has been much debate about the nature and aetiology of these cysts. Some appear to follow trauma (Taveras and Ransohoff, 1953) and others adhesive arachnoiditis (Horrax, 1924), but most remain unexplained.

During the investigation of patients with hydrocephalus in the Neurosurgical Department at The Hospital for Sick Children, there were 14 cases with an arachnoid cyst in the region of the subarachnoid cisterns or in the posterior fossa. In none of the cases had the presence of the cyst been suspected clinically. This paper reports the clinical, radiological, and operative findings in these cases.

A separate report deals in more detail with the radiological features in one of the cases (no. 9) (Strand and Hoare, in preparation).

\section{METHOD}

The case notes were studied for evidence of abnormalities in the pregnancy, delivery or early development that might be of aetiological significance, and

'Present address: The National Hospital for Nervous Diseases, Queen Square, London W.C.1. also for clinical features that might distinguish these $\vec{\circ}$ cases from others presenting with hydrocephalus. $\overrightarrow{\vec{\omega}}$ The neuroradiological investigations were reviewed $\stackrel{\omega}{\sigma}$ and in five cases it was possible to re-examine the histological material. The cases have been groupedo for the purposes of description according to the site $\omega_{+}^{\omega}$ of the cyst.

\section{GROUP I}

CYSTS OF CHIASMATIC OR INTERPEDUNCULAR CISTE⿺辶巛 (FIVE CASES) In these five patients hydrocephaluos $\subseteq$ was associated with evidence of basal cistern bloed and in addition in each a suprasellar cyst wa্े $\vec{\varphi}$ demonstrated. Three of the five showed ataxia of $\overrightarrow{ }$ gait or limbs and two had papilloedema. At follow-up two had poor vision with optic atrophy. Two had noo visual disturbance.

In most the cyst was large enough to block the foramen of Monro, and thereby contribute directly $\frac{\mathscr{Q}}{\mathrm{D}}$ to the hydrocephalus (in addition to the basal cistern $\stackrel{\square}{2}$ block). In four the hydrocephalus appeared to be $\overrightarrow{0}$ relieved by local drainage of the cyst however (see 3 discussion). Histologically, the cysts varied but in three there was glial tissue and an ependymal lining. These cannot be considered true cysts of the arachnoid therefore and may arise instead from glial cello rests (vide infra).

CASE 1

A 7 month old girl. The mother's pregnancy had been normal apart from mild toxaemia. The child was delivered after artificial rupture of the membranes, The neonatal 7 period was normal. At 4 months she had two episodes of vomiting and was limp and pale for 10 minutes on eachN occasion. When examined there was obvious hydrocephalus (skull circumference $49 \mathrm{~cm}$ ), a small sub- $\mathbb{O}$ occipital scalp haemangioma, and a palpable sacral spina స్ట bifida. 
Pneumoencephalography and fontanelle ventriculography showed dilated lateral and 3rd ventricles. The aqueduct and 4th ventricle were lifted up and were more horizontal in position than normal. The inferior part of the 3rd ventricle did not fill. A cyst filled below the 3rd ventricle, and appeared large enough to block the foramen of Monro. It extended into the sella and appeared to be located in the region of the chiasmatic cistern. Air failed to pass over the cortex suggesting a block in the basal cistern.

On 15 December 1967 a ventriculoatrial shunt was inserted. At follow-up just over two years later the skull circumference was $48.5 \mathrm{~cm}$ and the child was well.

\section{CASE 2}

A girl of $4 \frac{1}{2}$ years. Her mother had a normal pregnancy and delivery. The neonatal and developmental history was normal. Over a 10 month period she became progressively more unsteady. On examination the head circumference was $50.5 \mathrm{~cm}$ and there was bilateral papilloedema. Tone was increased in the limbs with mild right-sided weakness. Both plantar responses were extensor. There was slight ataxia of limbs and gross ataxia of gait.

Skull radiographs showed an enlarged sella turcica and a relatively short dorsum sellae, together with the changes of raised intracranial pressure. Ventriculography and pneumoencephalography showed dilated lateral and 3rd ventricles. There was a cavum septum pellucidum. The aqueduct was arched upwards and backwards. The 4th ventricle was small and was in the midline. There were several small parietal porencephalic cysts. A large suprasellar cavity filled with air and indented and lifted the floor of the 3rd ventricle. This cavity filled when the patient was supine and it was considered to be a cyst of the chiasmatic and interpeduncular cisterns filling from the pontine cistern. No air passed over the convexity of the hemispheres which suggested the presence of basal cistern block.

Ventricular drainage was established and at craniotomy (14 April 1967) a thin membrane was seen pouting forwards between the optic nerves. This membrane was opened releasing approximately $15 \mathrm{ml}$. clear fluid. An opening was also made into the 3rd ventricle with a further release of fluid. The arachnoid mater appeared thicker than normal. A piece of the membrane was examined histologically and found to consist of glial tissue with an ependymal lining.

Postoperatively, air from the cyst communicated freely with the 3rd ventricle. The child improved in alertness but ataxia of gait and the physical signs remained unchanged.

\section{CASE 3}

A boy of 20 months. His mother had a normal pregnancy and delivery. The neonatal and early history were normal. At 18 months he developed an ear infection and after this became unsteady. His mother noticed unsteadiness of his arms in reaching, and he stopped walking unassisted.

On examination, his head circumference was $51 \mathrm{~cm}$ and he had a head tilt to the left. There was bilateral ataxia on reaching and bilateral spasticity of the legs with extensor plantar responses.

A lumbar pneumoencephalogram showed dilated lateral and 3rd ventricles and a cavum septum pellucidum. The 4th ventricle was displaced backwards with stretching of the aqueduct. The interpeduncular cistern was lifted backwards and there was a filling defect in the floor of the 3rd ventricle. No air passed over the cerebral hemispheres.

At exploration on 7 September 1966 a pale cyst was found in the suprasellar region with two air bubbles visible through its wall. The foramen of Monro was dilated The cyst was deflated by aspiration and much of its wall removed. The histological report was of glial tissue and ependyma.

Postoperatively, he was able to walk, with less ataxia and spasticity. The plantar responses were now flexor. At follow-up three years later there was no ataxia or abnormality of tone.

\section{CASE 4}

A 14 month old boy had had a normal early history and had walked at 10 months. At 13 months he stopped walking, fell to the right while sitting and became irritable. It was suspected that his visual acuity had deteriorated.

On examination his head circumference was $50 \mathrm{~cm}$ with obvious hydrocephalus. He had bilateral papilloedema and a convergent strabismus of the right eye. The right middle ear was infected. There was bilateral ataxia on reaching and the reflexes were abnormally brisk.

Skull radiographs showed mastoiditis and the changes of increased intracranial pressure. Ventriculography and pneumoencephalography demonstrated dilated lateral and 3 rd ventricles. The aqueduct was pushed upwards and backwards. There was an indentation in the floor of the 3 rd ventricle. The $3 \mathrm{rd}$ ventricle was of different depths at different stages in the examination suggesting that the filling defect was due to a cyst in the region of the interpeduncular cistern. No air entered the pontine, interpeduncular, or chiasmatic cisterns, and none passed over the cerebral cortex, suggesting a block in the basal cisterns at the tentorial level (Fig. 1).

At operation (14 May 1969) a cyst with a bluish membrane was seen between the optic nerves, and the arachnoid mater appeared thickened. Ten millilitres of fluid were aspirated with collapse of the membrane. The lining was removed piecemeal. The cyst was thought at operation to resemble a craniopharyngioma. The histological appearance was, however, of strands of gliotic tissue with a desquamating ependymal type of epithelium on one surface, and connective tissue. There were some associated neurones and perivascular cuffs of glia.

A post-operative air study showed a reduction in the degree of hydrocephalus and a return of the 3rd ventricle to a more normal position. His convalescence was complicated by transient hypernatraemia and a left-sided fit. It became obvious that his vision was very poor. It has not been possible to follow his progress. 

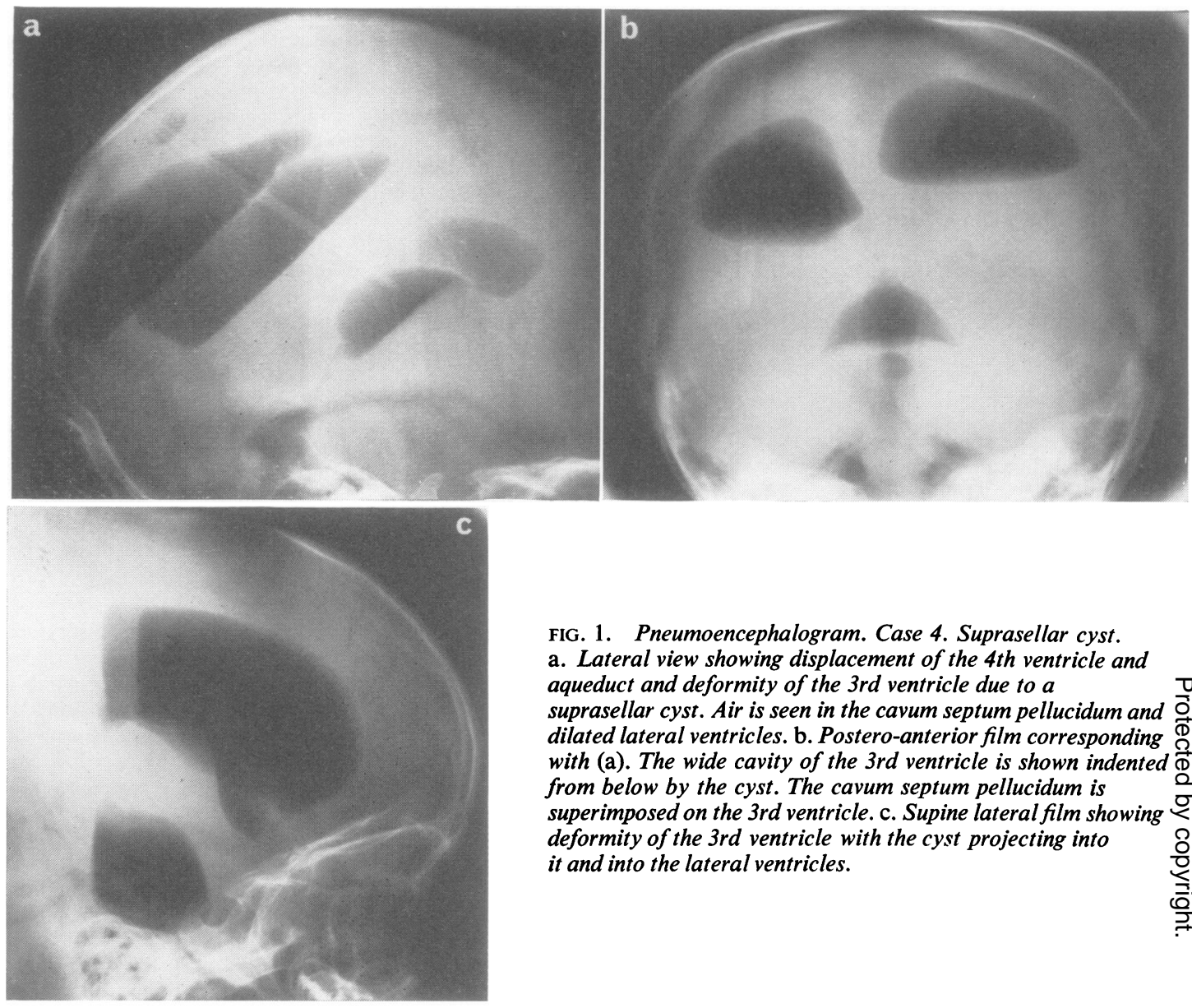

FIG. 1. Pneumoencephalogram. Case 4. Suprasellar cyst. a. Lateral view showing displacement of the 4th ventricle and aqueduct and deformity of the 3rd ventricle due to a suprasellar cyst. Air is seen in the cavum septum pellucidum and dilated lateral ventricles. b. Postero-anterior film corresponding with (a). The wide cavity of the 3rd ventricle is shown indented from below by the cyst. The cavum septum pellucidum is superimposed on the 3rd ventricle. c. Supine lateral film showing deformity of the 3rd ventricle with the cyst projecting into it and into the lateral ventricles.

\section{CASE 5}

A boy of $2 \frac{1}{2}$ years had had a normal medical history up to the age of 12 months. He began to pull himself up to stand but progressed no further in motor development in the second year, though speech development was probably normal. At $2 \frac{1}{2}$ years he began to 'bottom shuffle' and crawl with poor leg propulsion. He fed himself and played well with his hands.

On examination his head circumference was $52 \mathrm{~cm}$. He reached with some ataxia and sat well but would not stand. There was a curious shaking of his head and arms. The movements of the head were forwards and backwards with rotation and were not true 'bobble' movements.

A lumbar pneumoencephalogram showed dilated lateral ventricles. The 4 th ventricle was displaced backwards and the aqueduct was lifted and displaced backwards. There was a filling defect in the floor of the 3rd ventricle which varied in size during the examination. No air passed over the cerebral hemispheres.
At operation (12 March 1969) the arachnoid mater in the region of the optic chiasm appeared thickened. There $\mathbb{D}$ was an obvious suprasellar cyst which was punctured with the release of a large quantity of clear fluid. Free drainage 윽 of the cyst into the subarachnoid space was established. Histological examination of the cyst membrane showed only hyaline connective tissue.

Immediately postoperatively he showed less head movement and less ataxia but had a right third nerve palsy with probable reduction of visual acuity bilaterally. At followup 10 months later his head circumference was $53.75 \mathrm{~cm} .3$ His visual acuity appeared to be within normal limits and he stood with some help.

\section{GROUP II}

CYSTS OF THE QUADRIGEMINAL PLATE CISTERN (FOUR $\mathbb{N}$ CASES) All the patients in this group (cases 6 to 9), presented with hydrocephalus. None had ataxia but one had papilloedema, one optic atrophy, and a third $\omega$ 
was suspected of impaired visual acuity. Pneumography revealed evidence of basal cistern block and demonstrated in each case a space occupying lesion in the region of the quadrigeminal plate cistern. The operative findings confirmed the presence of a cyst in the two patients subjected to craniotomy. Histologically the cyst wall consisted of connective tissue only. Relief of the hydrocephalus followed removal of the cyst in one case, but in the other, a shunting procedure was also required.

\section{CASE 6}

A 15 month old boy had a normal early history. He failed to learn to walk with his twin and his mother noted enlargement of his head. On examination his head circumference was $48 \mathrm{~cm}$ and there was a slight increase of tone and reflexes on the left side.

A lumbar pneumoencephalogram showed dilated lateral ventricles. There was agenesis of the corpus callosum and a cyst in the right cerebral hemisphere communicating with the right lateral ventricle. The aqueduct was displaced downwards and forwards, suggesting the presence of a cyst in the region of the quadrigeminal plate cistern. Air passed over the cerebral cortex and outlined normal cortical sulci. In view of the porencephalic cyst and agenesis of the corpus callosum no surgery was attempted.

At the age of 9 years he was not doing well at school and had a mild left hemiparesis. He was reinvestigated by ventriculography which confirmed the earlier features and showed that the space occupying lesion behind and above the aqueduct was a cyst. At the latest follow-up he was having occasional left-sided fits and had persistent enuresis.

\section{CASE 7}

A 4 month old girl was born three weeks prematurely in a pregnancy complicated by oedema and albuminuria. She had neonatal jaundice with a serum bilirubin reaching a peak of $17 \mathrm{mg} / 100 \mathrm{ml}$. At $2 \frac{1}{2}$ months there was obvious enlargement of the skull and a 'setting sun' appearance of the eyes.

On examination the head circumference was $46.5 \mathrm{~cm}$ and the baby did not appear to react to light or follow objects.

A lumbar pneumoencephalogram showed dilated lateral and 3rd ventricles. The aqueduct was lifted and displaced forwards. The ambiens cisterns were dilated and no air passed over the cerebral hemispheres, suggesting a block in the basal cisterns at the tentorial orifice. There was in addition a small frontal porencephalic cyst. The forward displacement of the aqueduct was thought to be due to a cyst of the quadrigeminal plate cistern.

A ventriculoatrial shunt was inserted (14 October 1966) and later revised. The infant had, however, severe brain damage, and the prognosis was considered hopeless. She died aged 7 months.

\section{CASE 8}

A girl aged 3 months. Her mother had had a normal pregnancy and delivery. The child then developed a progressive increase in head circumference and vomited after feeds. On examination there was marked hydrocephalus (skull circumference $45.5 \mathrm{~cm}$ ). There was thought to be mild optic pallor.

Combined ventriculography and pneumoencephalography demonstrated large lateral ventricles and a large cisterna magna. The ambiens cisterns were dilated. The 4th ventricle was compressed and pushed forwards. The posterior end of the 3 rd ventricle was displaced forwards. Air introduced by the lumbar route failed to pass through the aqueduct. No air passed from the basal cisterns over the cerebral cortex. It was concluded that there was obstruction of the aqueduct due to a space occupying lesion deforming the posterior end of the 3rd ventricle and the junction of the 3rd ventricle and aqueduct.

At exploration (16 August 1967) a large bluish cyst was found in the quadrigeminal cistern. Clear fluid $(25 \mathrm{ml}$.) was aspirated. A rubber catheter was left in the cyst to drain it into the posterior fossa.

Postoperatively the 'setting sun' appearance persisted and she had mild bilateral optic atrophy.

\section{CASE 9}

A 9 month old boy. His mother had had a normal pregnancy and delivery. The neonatal period was normal. At 8 weeks of age his mother noted that his eyes tended to drop showing the upper sclerae. At 9 months he was still not lifting his head or sitting. On examination his head circumference was $52.5 \mathrm{~cm}$. There was obvious hydrocephalus with a 'sunset' appearance of the eyes. There was bilateral papilloedema. The limbs appeared normal.

A fontanelle ventriculogram showed dilated lateral and 3 rd ventricles. The aqueduct was vertically aligned and the 4th ventricle was displaced anteriorly. The posterior end of the 3rd ventricle was indented and pushed upwards with a thinned tilted suprapineal recess. The pontine cistern was flattened. The interpeduncular cistern was indented by the enlarged 3rd ventricle. No air passed over the hemispheres suggesting a basal cistern block.

A posterior fossa exploration was carried out ( 9 November, 1966) at which a bluish cyst was seen above the vermis and separating the cerebellar hemispheres. The cyst extended through the tentorial opening and was causing brain-stem compression. The cyst was punctured and part of its wall removed. Histological examination of the wall revealed loose connective tissue only.

A postoperative pneumoencephalogram (Fig. 2) showed persistent deformity of the posterior wall of the 3 rd ventricle, aqueduct, and roof of the 4 th ventricle indicative of the presence of the cyst in the posterior part of the tentorial hiatus.

Postoperatively, the child remained drowsy and so a ventriculoatrial shunt was inserted. His drowsiness was relieved but he developed left-sided seizures. Eight months later, aged 17 months, he was mentally retarded with bilateral increase in tone in the limbs. 


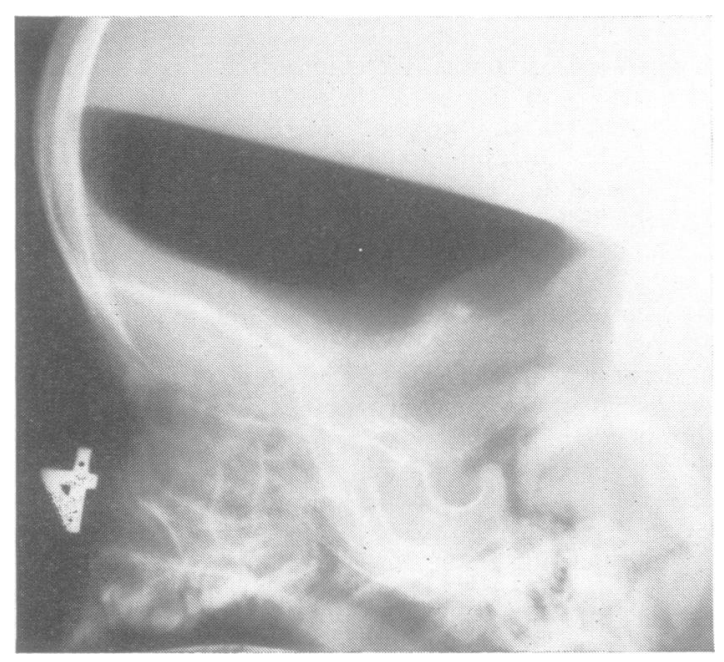

FIG. 2. Postoperative pneumoencephalogram. Case 9. Quadrigeminal plate cyst. Lateral view showing dilated lateral and 3rd ventricles, and deformity of the posterior end of the 3rd ventricle, aqueduct, and roof of the 4th ventricle, due to the presence of a cyst in the posterior part of the tentorial hiatus.

\section{GROUP III}

CYSTS IN THE POSTERIOR FOSSA (FIVE CASES) In this group (cases 10 to 14 ) the presenting problem was again hydrocephalus. Two showed some ataxia but in none was a posterior fossa lesion confidently diagnosed clinically. Air studies revealed a space occupying lesion in the posterior fossa in three cases. Even at exploration it was not always possible to determine the site of origin of the cyst. In two cases the cyst wall consisted of glia and flattened epithelium. In a third it strongly resembled medullary velum and this case (case 11) may be an example of a diverticulum of the 4 th ventricle as seen in the Dandy-Walker syndrome.

\section{CASE 10}

A boy of 2 years 9 months. His mother had a normal pregnancy and delivery. The neonatal period was normal. At 2 months, 4 days after vaccination, he became lethargic with a tense fontanelle and 'setting sun' appearance of the eyes. He was unwell for one month and then recovered. His development proceeded normally. Three months before admission he began to complain of head pains and to vomit. On examination he was dull and unresponsive with a skull circumference of $54.75 \mathrm{~cm}$. Visual acuity appeared to be normal. His gait was slightly unsteady.
A ventriculogram showed dilated lateral and $3 \mathrm{r} \propto$ ventricles. The aqueduct was kinked at its origin. The 4th ventricle was displaced to the right. No air passed over? the hemispheres. A left-sided cerebellar hemisphere lesiono was diagnosed and the posterior fossa explored (20 Maye 1953). The left cerebellar hemisphere was pushed to the left by a large cyst containing clear fluid. The cyst wall was found to consist of a thin strand of glial tissue with a layer of flattened cells on one surface. A postoperative ventricu $=$ logram showed a reduction in the size of the ventricles? Fifteen months after surgery he was noted to have slight. limb ataxia but thereafter progressed well. He wrote a the age of 19 years in September 1969 reporting that he? was well, and at university.

\section{CASE 11}

A 5 month old boy had had a normal early history anf appeared to be developing normally but his head enes larged too rapidly and his eyes were depressed. On $\vec{b}$ examination his head circumference was $56 \mathrm{~cm}$. The limbs were normal.

A ventriculogram showed dilated lateral and $3 \mathrm{rdp}$ ventricles with a large filling defect in the 4th ventricle Air passed into the basal cisterns but no air outlined the cortical sulci. At operation (21 February 1964) a cyst was w found compressing the cerebellum forwards. A partia removal was carried out opening the cyst into wiew cisterna magna.

Histological sections showed that the cyst wall căb sisted of connective tissue, calcified debris and arachnote cells. On the inner surface there was a cuboidal cell la er resembling ependyma, and on the other surface abnorraa cerebellar cortex was recognizable with granule ceds,, Purkinje cells, and a molecular layer. There was wife $\overrightarrow{0}$ spread gliosis.

Postoperatively the intracranial pressure remained high and so a ventriculoatrial shunt was inserted. At this second operation the occipital wound broke down causing a precipitate fall in intracranial pressure and producing bilateral subdural haematomata. Despite drainage of the subdural spaces he remained severely damaged.

\section{CASE 12}

A 3 year old boy had been noticed at birth to have a large head (circumference $38 \mathrm{~cm}$ ). At 14 months he was. referred as he was not crawling or standing and his heaff circumference was then $55 \mathrm{~cm}$. He was found to be mentally retarded and he was followed up as an out -5 patient. At the age of 3 years he began to vomit and was admitted stuporose with a head circumference of $60 \mathrm{~cm}$.

A ventricular drain was inserted. Ventriculography? showed greatly dilated ventricles with a shift to the lef 2 of the lateral ventricles. There was a large right hemi-o sphere cyst. The pontine cistern was flattened. The $4 \mathrm{th}$ ventricle was compressed from above. Air outlined theo cerebral sulci. At operation (23 February 1962) a large cyst was found in the middle cranial fossa on the right o Arising from the tentorial orifice was a second cyst lying alongside the midbrain. This cyst contained pale yellow fluid. It was opened widely so that it communicatedw 
freely with the cyst in the middle fossa. A postoperative pneumoencephalogram showed less shift and air now passed through the tentorial opening. He was more alert but remained retarded. At follow-up $2 \frac{1}{2}$ years later he was having occasional seizures and seemed to have a mental age of about 3 to 5 years (chronological age 7 years).

\section{CASE 13}

A girl of $4 \frac{1}{2}$ months had been $4 \mathrm{lb} .11 \mathrm{oz}(2 \cdot 125 \mathrm{~kg})$ at birth. Throughout the first few months of life her head size enlarged at an abnormal rate and it was noted that she could follow objects to either side but not up and down. She was unable to roll her head on the pillow or support it. On examination there was obvious hydrocephalus (circumference $49 \mathrm{~cm}$ ).

A fontanelle ventriculogram showed dilated lateral and 3 rd ventricles. The 4 th ventricle was small and displaced anteriorly. The cisterna magna was large. The cerebellopontine angle cisterns were dilated and air passed from them into a space below the suprapineal recess of the 3rd ventricle. No air entered the interpeduncular or chiasmatic cisterns, and none outlined the cerebral sulci. There was thought to be basal cistern block at the tentorial opening with dilated posterior fossa cisterns (Fig. 3).

At operation (18 February 1960) a large midline cystic cavity was found overlying the cerebellum. The cerebellum, medulla, and upper cervical cord all appeared small. In the subtentorial region the cyst was seen to be compressing the brain-stem anteriorly and obstructing the tentorial opening. It was not in continuity with the cisterna magna below. A connection was made between the extensive cyst and the cisterna magna, but it did not seem likely that the tentorial block would be relieved.
Postoperatively an occipital meningocoele developed. The child made little progress and 7 months later the head circumference had increased to $62 \mathrm{~cm}$.

CASE 14

A $5 \frac{1}{2}$ year old girl had been noted to have a large head at birth. Her motor milestones were a little delayed and from the age of 4 years it had been thought that she was becoming unsteady on her feet. On examination she was mildly retarded with a head circumference of $60 \mathrm{~cm}$. There was mild bilateral ataxia of the limbs.

A ventriculogram showed dilated lateral and 3rd ventricles. The 4 th ventricle was displaced forwards and slightly to the left. No air passed over the cerebral hemispheres. A right-sided posterior fossa spaceoccupying lesion was diagnosed.

At operation (14 March 1956) a cyst was found adherent at the tentorial orifice on the right side between the brainstem and the tentorium cerebelli. The cyst was opened and found to communicate with the cavity of the 4th ventricle Histologically, the cyst wall consisted of neuroglia and a single layer of flattened cells.

At follow up two years later she was doing well at school and had no abnormal physical signs.

\section{DISCUSSION}

All the cases in this series presented with hydrocephalus. Five showed some ataxia of limbs or gait and as only nine were of an age where ataxia could be convincingly demonstrated (over 1 year old) this must be considered a common feature. Abnormalities
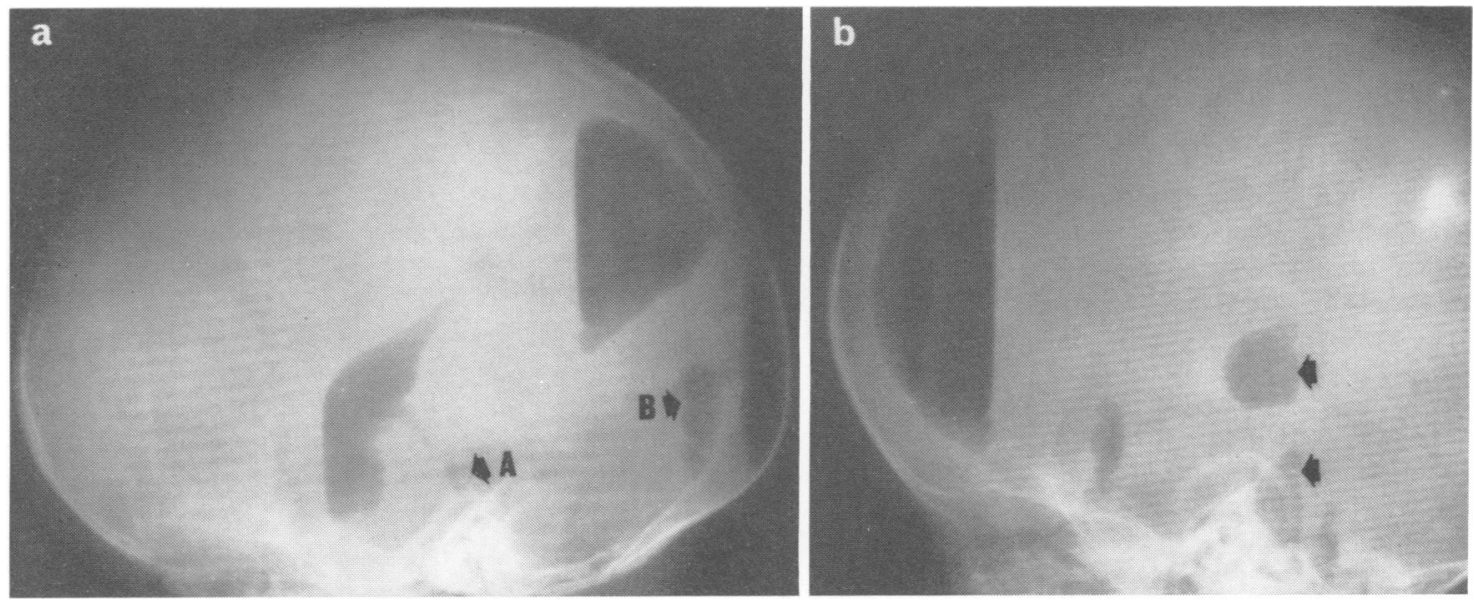

FIG. 3. Ventriculogram. Case 13. Posterior fossa cyst.

a. Lateral view showing a dilated 3rd ventricle which is generally displaced upwards and forwards. The suprapineal recess is flattened. The aqueduct is displaced anteriorly and the small 4th ventricle $(A)$ is displaced forwards. Air has escaped into the cisterna magna which is large and into a cavity $(B)$. b. The anterior extent of the cavity $B$ is shown by the upper arrow. Air is seen in a dilated cerebellopontine angle cistern (lower arrow). 
of vision were also common, affecting seven of the children. Cysts in the suprasellar region were particularly associated with abnormalities of vision and ataxia (three out of five). These two features may be more common than in cases of hydrocephalus without associated cysts, but the series is too small for conclusions to be drawn. No other clinical features distinguished these cases.

Pneumography demonstrated hydrocephalus and, in all but one case, the features of basal cistern block. The cysts in the suprasellar region caused a filling defect in the floor of the 3 rd ventricle. They were frequently large enough to be capable of obstructing the foramina of Monro. When the filling defect in the floor of the 3 rd ventricle varied in size during the investigation or itself filled with air, it was possible preoperatively to demonstrate that the lesion was a cyst.

In the region of the quadrigeminal plate cistern, cysts caused displacement of the aqueduct downwards and forwards and a shift forwards of the posterior end of the 3rd ventricle. These features were described by Lourie and Berne (1961) and considered diagnostic. They were also noted by Kruyff (1965). The differential diagnosis includes pinealoma and aneurysms of the vein of Galen which may be distinguished by angiography (Kruyff, 1965).

In the case of the posterior fossa cysts, it was not possible on air studies to distinguish them from other space-occupying lesions.

In all of the cases save one the hydrocephalus was associated with and attributed to basal cistern block (Dandy, 1921). It has been suggested that some cysts in the subarachnoid region form by the separation of the two layers of arachnoid mater at a time when the subarachnoid space is developing (Starkman et al., 1958). It would therefore seem reasonable for cyst formation to occur in parallel with the production of basal cistern block, as both would represent maldevelopment of the subarachnoid space. Some of the cysts whose walls consisted of connective tissue or leptomeningeal tissue only, may have formed in this way. The majority of the present cases, however, were found histologically to consist of glial tissue with an ependymal-like lining. A simple loculation or interarachnoid collection of fluid cannot explain these cysts. It seems more likely that they arise as separate but associated developmental abnormalities. Rests of glial tissue have been found in the subarachnoid tissue, particularly around the base of the brain (Cooper and Kernohan, 1951), and these cell rests may be the source of the cysts.

Other features support the idea of a developmental origin. There was no evidence of neonatal haemorrhage, infection, or trauma in these cases, and many showed other signs of maldevelopment. Thus porencephalic cysts and agenesis of the corpus callosum were encountered in this group.

In the posterior fossa the origin of the cysts if probably more variable. Cysts of the cerebelloo pontine angle cistern have been described an attributed to atresia of the foramina of Lushka with the separation of the embryonal double layer as $\mathscr{E}$ cyst (Gardner, McCormack, and Dohn, 1960). The Dandy-Walker syndrome consists of hydrocephalus due to obstruction to outflow of cerebrospinal fluid: in the foramina of the 4th ventricle. A dilatation of the 4th ventricle occurs producing a pseudocyst in the posterior fossa (D'Agostino, Kernohan, ant Brown, 1963). The wall of the pseudocyst consists of ependyma, glia, and heterotopic cerebellar tissue. IF resembles the normal structure of medullary velun 9 from which it arises. Case 11 of the present series̈ shows this histological appearance and is therefors likely to be an example of a diverticulum of the 4th ventricle. One other case showed continuity between $\vec{w}$ a cyst and the 4th ventricle. Alvord (1961) describes? a case with similar histological findings to case 11 and suggested that the cyst arose from a folium of the cerebellum as an internal meningoencephalocoele

In the present series local drainage and openingof the cysts often appeared to control the hyd官 $\vec{\sigma}$ cephalus. This suggests that the cysts had contributed directly to the block in the cerebrospinal fluid path? ways and many were in fact large enough to have caused some degree of obstruction in the aqueducbo foramina of Monro. There was however evidenceeof basal cistern block in all but one case. This bl was presumably often incomplete as not all of cases required a shunt procedure.

It is recommended when cysts of this type are found in infants with hydrocephalus, that they ars first explored and then that postoperative pneumo encephalography is carried out to assess whether ard additional shunting procedure is required.

It is a pleasure to express my gratitude to $\mathrm{Mr}$. Kenneth Till, Dr. R. D. Hoare, and Dr. J. Wilson for their hel and encouragement in the preparation of this paper. Dr. A. Dayan kindly reviewed the histological materiat described.

\section{REFERENCES}

Alexander, E. (1953). Benign subtentorial supracollicular cys as a cause of obstructive hydrocephalus. J. Neurosurg., 10 317-323.

Alvord, E. C. (1961). The pathology of hydrocephalus. In Disorders of the Developing Nervous System, pp. 343-419Edited by W. S. Fields and M. M. Desmond. Thomas Springfield, Illinois.

Alvord, E. C., and Marcuse, P. M. (1962). IntracraniaP cerebellar meningo-encephalocele (posterior fossa cyst $N$ causing hydrocephalus by compression at the incisurat tentorii. J. Neuropath. exp. Neurol., 21, 50-69. 
Anderson, F. M., and Landing, B. H. (1966). Cerebral arachnoid cysts in infants. $J$. Paediat., 69, 88-96.

Cooper, I. S., and Kernohan, J. W. (1951). Heterotopic glial nests in the subarachnoid space: histopathologic characteristics, mode of origin and relation to meningeal gliomas. $J$. Neuropath exp. Neurol., 10, 16-29.

D'Agostino, A. N., Kernohan, J. W., and Brown, J. R. (1963). The Dandy-Walker syndrome. J. Neuropath exp. Neurol., 22, 450-470.

Dandy, W. E. (1921). The cause of so-called idiopathic hydrocephalus. Bull. Johns Hopk. Hosp., 32, 67-75.

Dott, N. M., and Gillingham, F. J. (1958). Mechanical aspects of the cerebrospinal fluid circulation-physiological pathological, surgical. In The Cerebrospinal Fluid. 246-264. Edited by G. E. W. Wolstenholme and C. M. O'Connor. Churchill: London.

Gardner, W. J., McCormack, L. J., and Dohn, D. F. (1960). Embryonal atresia of the fourth ventricle. The cause of 'arachnoid cyst' of the cerebellopontine angle. J. Neurosurg., 17, 226-237.

Horrax, G. (1924). Generalized cisternal arachnoiditis simulating cerebellar tumour: Its surgical treatment and end-results. Arch. Surg., 9, 95-112.
Jakubiak, P., Dunsmore, R. H., and Beckett, R. S. (1968). Supratentorial brain cysts. J. Neurosurg., 28, 129-136.

Katagiri, A. (1960). Arachnoidal cyst of the cisterna ambiens. Neurology (Minneap.), 10, 783-786.

Kruyff, E. (1965). Paracollicular plate cysts. Amer. J. Roentgenol., 95, 899-916.

Lourie, H., and Berne, A. S. (1961). Radiological and clinical features of an arachnoid cyst of the quadrigeminal cistern. J. Neurol. Neurosurg., Psychiat., 24, 374-378.

Matson, D. D. (1969). Neurosurgery of Infancy and Childhood, 2nd ed. Thomas: Springfield, Illinois.

Starkman, S. P., Brown, T. C., and Linell, E. A. (1958). Cerebral arachnoid cysts. J. Neuropath. exp. Neurol., 17, 484-500.

Strand, R. D., and Hoare, R. D. Subarachnoid fluid collections in hydrocephalus. In preparation.

Taveras, J. M., and Ransohoff, J. (1953). Leptomeningeal cysts of the brain following trauma with erosion of the skull. J. Neurosurg., 10, 233-241.

Trowbridge, W. V., and French, J. D. (1952). Benign arachnoid cysts of the posterior fossa. J. Neurosurg., 9 , 398-404. 